\title{
Magnon Heat Conductivity and Mean Free Paths in Two-Leg Spin Ladders: A Model-Independent Determination
}

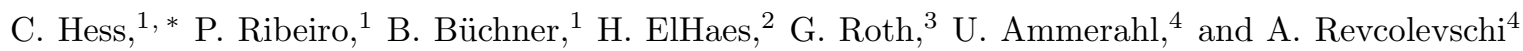 \\ ${ }^{1}$ IFW-Dresden, Institute for Solid State Research, P.O. Box 270116, D-01171 Dresden, Germany \\ 22. Physikalisches Institut, RWTH-Aachen, 52056 Aachen, Germany \\ ${ }^{3}$ Institut für Kristallographie, RWTH-Aachen, 52056 Aachen, Germany \\ ${ }^{4}$ Laboratoire de Physico-Chimie de l'État Solide, Université Paris-Sud, 91405 Orsay, France
}

(Dated: November 21, 2018)

\begin{abstract}
The magnon thermal conductivity $\kappa_{\text {mag }}$ of the spin ladders in $\mathrm{Sr}_{14} \mathrm{Cu}_{24-\mathrm{x}} \mathrm{Zn}_{\mathrm{x}} \mathrm{O}_{41}$ has been investigated at low doping levels $x=0,0.125,0.25,0.5$ and 0.75 . The Zn-impurities generate nonmagnetic defects which define an upper limit for $l_{\text {mag }}$ and therefore allow a clear-cut relation between $l_{\text {mag }}$ and $\kappa_{\text {mag }}$ to be established independently of any model. Over a large temperature range we observe a progressive suppression of $\kappa_{\text {mag }}$ with increasing $\mathrm{Zn}$-content and find in particular that with respect to pure $\mathrm{Sr}_{14} \mathrm{Cu}_{24} \mathrm{O}_{41} \kappa_{\text {mag }}$ is strongly suppressed even in the case of tiny impurity densities where $l_{\text {mag }} \lesssim 374 \AA$. This shows unambiguously that large $l_{\text {mag }} \approx 3000 \AA$ which have been reported for $\mathrm{Sr}_{14} \mathrm{Cu}_{24} \mathrm{O}_{41}$ and $\mathrm{La}_{5} \mathrm{Ca}_{9} \mathrm{Cu}_{24} \mathrm{O}_{41}$ on basis of a kinetic model are in the correct order of magnitude.
\end{abstract}

\section{INTRODUCTION}

The transport of energy and magnetization in low dimensional quantum magnets is subject to numerous experimental and theoretical investigations 1.2 .3 .4 .5 .6 .7 .8 .9 .10 .11 .12 .13 .14 .15 .16 .17 .18 .19 .20 .21 .22 .23$. with the nature of scattering and dissipation of thermal and magnetic currents at the center of interest. In particular, quasi one-dimensional (1D) systems are being intensely studied since properties starkly different from classical systems are expected: ballistic magnetic heat transport, i.e. a finite thermal Drude weight $D_{\mathrm{th}}$, is predicted in integrable models like the $X X Z$ Heisenbergchain with $S=1 / 2.10,12,13,14,15,24$ Experimentally, for the $S=1 / 2$ two-leg spin ladder systems $\mathrm{Sr}_{14} \mathrm{Cu}_{24} \mathrm{O}_{41}$ and $\mathrm{La}_{5} \mathrm{Ca}_{9} \mathrm{Cu}_{24} \mathrm{O}_{41}$ exceptionally large values of the magnetic thermal conductivity $\kappa_{\text {mag }}$ are found (up to $\sim 100 \mathrm{Wm}^{-1} \mathrm{~K}^{-1}$ even at room temperature) ${ }^{1,2,3,4,5} \mathrm{In}$ principle, these data should provide the unique possibility of extracting the magnetic mean free path $l_{\text {mag }}$, hence obtaining information about magnetic scattering processes in such low-dimensional spin systems for the first time. It turned out, however, that the extracted values of $l_{\text {mag }}$ are strongly model dependent: on one hand, the analysis within the framework of a simple kinetic model yielded values up to $l_{\text {mag }} \approx 3000 \AA \AA^{2}$ For the complicated and supposedly rather imperfect materials studied such values are surprisingly large and might arise from oversimplifications inherent to the model. On the other hand, much smaller values were obtained in a more sophisticated analysis which is based on a finite $D_{\text {th }}{ }^{11}$ However, the finiteness of $D_{\text {th }}$ in these systems is subject of a highly controversial debate since both ballistic and non-ballistic heat transport has been predicted for non-integrable $S=1 / 2$ models such as these two-leg spin-ladders or frustrated chains, $11,14,15,16,19,20,21,23$.

In this article we present an investigation of $\kappa_{\text {mag }}$ and $l_{\text {mag }}$ of $\mathrm{Sr}_{14} \mathrm{Cu}_{24} \mathrm{O}_{41}$ which is independent of any model. The basic idea is to generate nonmagnetic defects in the material and thereby impose an upper limit for $l_{\text {mag }}$ in a well-defined manner. We achieved this by substituting small amounts of nonmagnetic $\mathrm{Zn}^{2+}$ for the magnetic $\mathrm{Cu}^{2+}$ (with $S=1 / 2$ ). $\kappa_{\text {mag }}$ of $\mathrm{Sr}_{14} \mathrm{Cu}_{24-\mathrm{x}} \mathrm{Zn}_{\mathrm{x}} \mathrm{O}_{41}$ $(x \leq 0.75)$ is progressively suppressed with increasing $\mathrm{Zn}$ 2425 tent and, in particular, already at tiny impurity densities ylelding $l_{\text {mag }} \lesssim 374 \AA$ this suppression is strong with respect to the pure material (exceeding a factor of $\sim 5$ ). Employing the simple kinetic model which had been used in previous studies 2.5 we even find $l_{\text {mag }} \approx d_{\mathrm{Zn}-\mathrm{Zn}}$ at low temperatures, where $d_{\mathrm{Zn}-\mathrm{Zn}}$ is the nominal mean distance between Zn-ions along a ladder. This unambiguously shows that the large $l_{\text {mag }} \approx 3000 \AA$ which have been reported for $\mathrm{Sr}_{14} \mathrm{Cu}_{24} \mathrm{O}_{41}$ and $\mathrm{La}_{5} \mathrm{Ca}_{9} \mathrm{Cu}_{24} \mathrm{O}_{41}$ are in the correct order of magnitude.

\section{EXPERIMENTAL}

We have grown single crystals of $\mathrm{Sr}_{14} \mathrm{Cu}_{24-\mathrm{x}} \mathrm{Zn}_{\mathrm{x}} \mathrm{O}_{41}$ $(x=0,0.125,0.25,0.5,0.75)$ by the travelling solvent floating zone method 27 The thermal conductivity as a function of temperature $T$ of these crystals has been measured with a focus on the crystallographic $c$-direction $\left(\kappa_{c}\right)$, which is parallel to the orientation of the ladders 34 The measurements in the range $7-300 \mathrm{~K}$ have been performed with a standard four probe technique ${ }^{2.28 .29}$

\section{RESULTS AND ANALYSIS}

Fig. 11 a) shows the temperature dependence of the thermal conductivity of the undoped material, i.e. $\mathrm{Sr}_{14} \mathrm{Cu}_{24-\mathrm{x}} \mathrm{Zn}_{\mathrm{x}} \mathrm{O}_{41}$ at $x=0$ along all three crystal axes. In this material the thermal conductivity along the ladder direction, $\kappa_{c}(T)$, frequently displays a double peak structure. 1.2 .3 .4 .5 The low-temperature peak $(\sim 20 \mathrm{~K})$ is observed along all crystal directions and arises due to the usual phonon heat conduction. The peak at higher tem- 


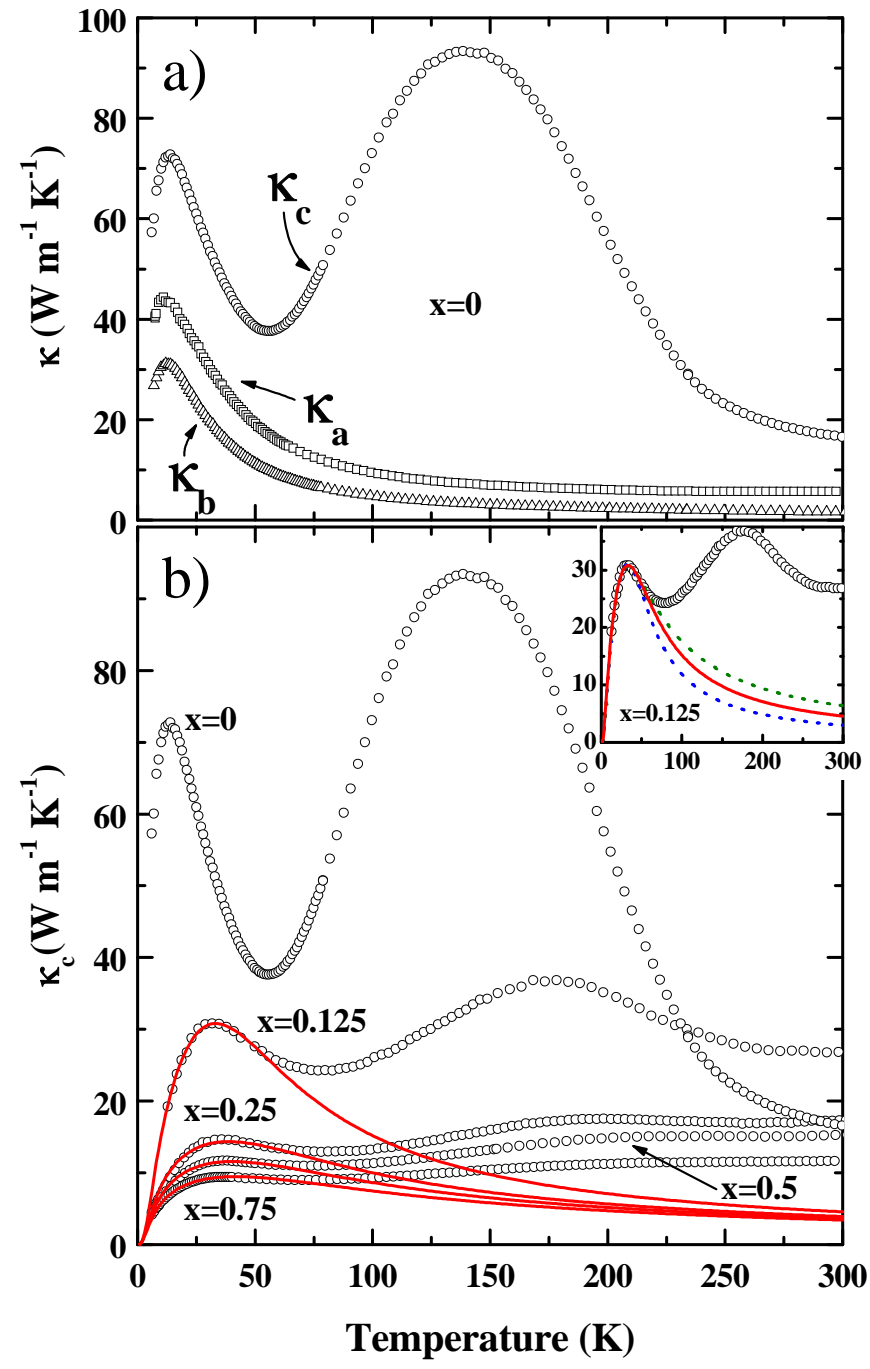

FIG. 1: Thermal conductivity of $\mathrm{Sr}_{14} \mathrm{Cu}_{24-\mathrm{x}} \mathrm{Zn}_{\mathrm{x}} \mathrm{O}_{41}(x=$ $0,0.125,0.25,0.5,0.75)$ as a function of temperature. a) Anisotropic thermal conductivity along $a^{-}, b$-, and $c$-axes $\left(\kappa_{a}\right.$, $\kappa_{b}, \kappa_{c}$ ) at $x=0$ (cf. Ref. 25). b) $\kappa_{c}$ at $x \geq 0$. Solid lines represent the estimated phononic background of $\kappa_{c}$ (cf. text). Inset: Estimations for the uncertainties of the phonon background in the representative example $\kappa_{c}$ at $x=0.125$ (dotted lines).

perature, however, is only observed along the $c$-axis and is the signature of strictly one-dimensional magnetic heat conduction along the ladders in the material 1.2 In the following we concentrate on the evolution of $\kappa_{c}$ at finite $\mathrm{Zn}$

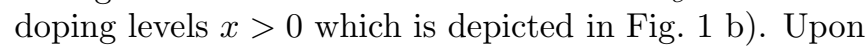
Zn-doping, $\kappa_{c}$ is progressively suppressed and exhibits less pronounced peaks. The magnetic peak simultaneously broadens and apparently shifts towards higher temperature since the peak structure evolves into a monotonic increase at high temperature.

Qualitatively, the doping induced suppression of both phononic and magnetic transport channels of $\kappa_{c}$ is a natural consequence of the induced structural and magnetic defects: different constituents on the same lattice site $(\mathrm{Cu}$ and Zn) must lead to enhanced scattering of phonons. Similarly, since the $\mathrm{Zn}^{2+}$ ions create nonmagnetic sites in a $S=1 / 2$ spin ladder built of predominantly $\mathrm{Cu}^{2+}$ ions, magnetic excitations scatter on these sites. The upper limits for $l_{\text {mag }}$ thus created correspond to the mean distances between a pair of $\mathrm{Zn}$-ions along a ladder, i.e. $d_{\mathrm{Zn}-\mathrm{Zn}} \approx 374 \AA, 187 \AA, 94 \AA$ and $62 \AA$. Apparently, even the small Zn-contents discussed here lead to an efficient suppression of $\kappa_{\text {mag }}$ with respect to the undoped material. We can therefore conclude without further analysis that $l_{\text {mag }}$ of pure $\mathrm{Sr}_{14} \mathrm{Cu}_{24} \mathrm{O}_{41}$ must be significantly larger than the highest $d_{\mathrm{Zn}-\mathrm{Zn}} \approx 374 \AA$.

Our aim is now to analyze the magnetic heat conductivity $\kappa_{\text {mag }}$ of the ladders in more detail; the phononic contribution $\kappa_{\mathrm{ph}}$ to $\kappa_{c}$ must therefore be estimated and subtracted from $\kappa_{c}$. The estimation of $\kappa_{\mathrm{ph}}$ is based on the fact that due to the large spin gap of order $\sim 400 \mathrm{~K}, 30.31 \kappa_{\mathrm{mag}}$ is negligibly small at $T \lesssim 50 \mathrm{~K}$ and hence $\kappa_{\mathrm{ph}} \approx \kappa_{c}$ in this temperature range. Therefore, the usual procedure ${ }^{1.2 .5}$ is to fit $\kappa_{c}$ at $T \lesssim 50 \mathrm{~K}$ with a Debye mode $^{32}$ and then extrapolate the fit function towards higher temperature. In our analysis of $\kappa_{\mathrm{ph}}$ in the $\mathrm{Zn}$ doped compounds we impose the additional constraint of identical fit parameters for all doping levels provided that they are not related to phonon-defect scattering. The coefficient for phonon-defect scattering is allowed to vary with increasing $\mathrm{Zn}$-content ${ }^{35}$ and the results of this procedure are shown in Fig. (1) b) as solid lines. Such an extrapolation of $\kappa_{\mathrm{ph}}$ is certainly not exempt from uncertainty. We take these into account by estimating positive and negative deviations from $\kappa_{\mathrm{ph}} \stackrel{36}{\underline{3}}$ A representative example of such deviation is shown in the inset of Fig. 1 for $x=0.125$.

Fig. 22 displays the resulting $\kappa_{\text {mag }}$ of the four Zn-doped compounds as a function of temperature. The peak-like shape apparent in the curve at $x=0.125$ reflects the typical temperature dependence of the thermal conductivity of a system with a moderate number of impurities: at low temperature, where scattering rates are only weakly temperature dependent, the strong increase in the number of quasiparticles generates an increasing $\kappa$. The decrease of $\kappa$ at intermediate temperature $(180 \mathrm{~K} \lesssim T \lesssim 250 \mathrm{~K})$ and the almost constant to slightly increasing $\kappa$ at further rising temperature signal a strong increase of the scattering rate and a weakening or even saturation of this increase, respectively. The relevant scattering processes for $\kappa_{\text {mag }}$ in the undoped compound $\mathrm{Sr}_{14} \mathrm{Cu}_{24} \mathrm{O}_{41}$ are scattering on defects at low temperature and magnon-hole scattering at intermediate and high temperatures ${ }^{4.5}$ In the case of our weakly doped samples with a Zn-concentration $\lesssim 3 \%$ it is reasonable to assume that this situation is in principle preserved with the particularity, however, that the defects relevant for the magnon scattering are mainly defined by the Zn-dopants. In this scenario one expects the temperature independent magnon-defect scattering to increasingly dominate over the temperature dependent magnon-hole scattering, when the Zn-doping, i.e. the density of defects is further increased. $\kappa_{\text {mag }}$ evolves 


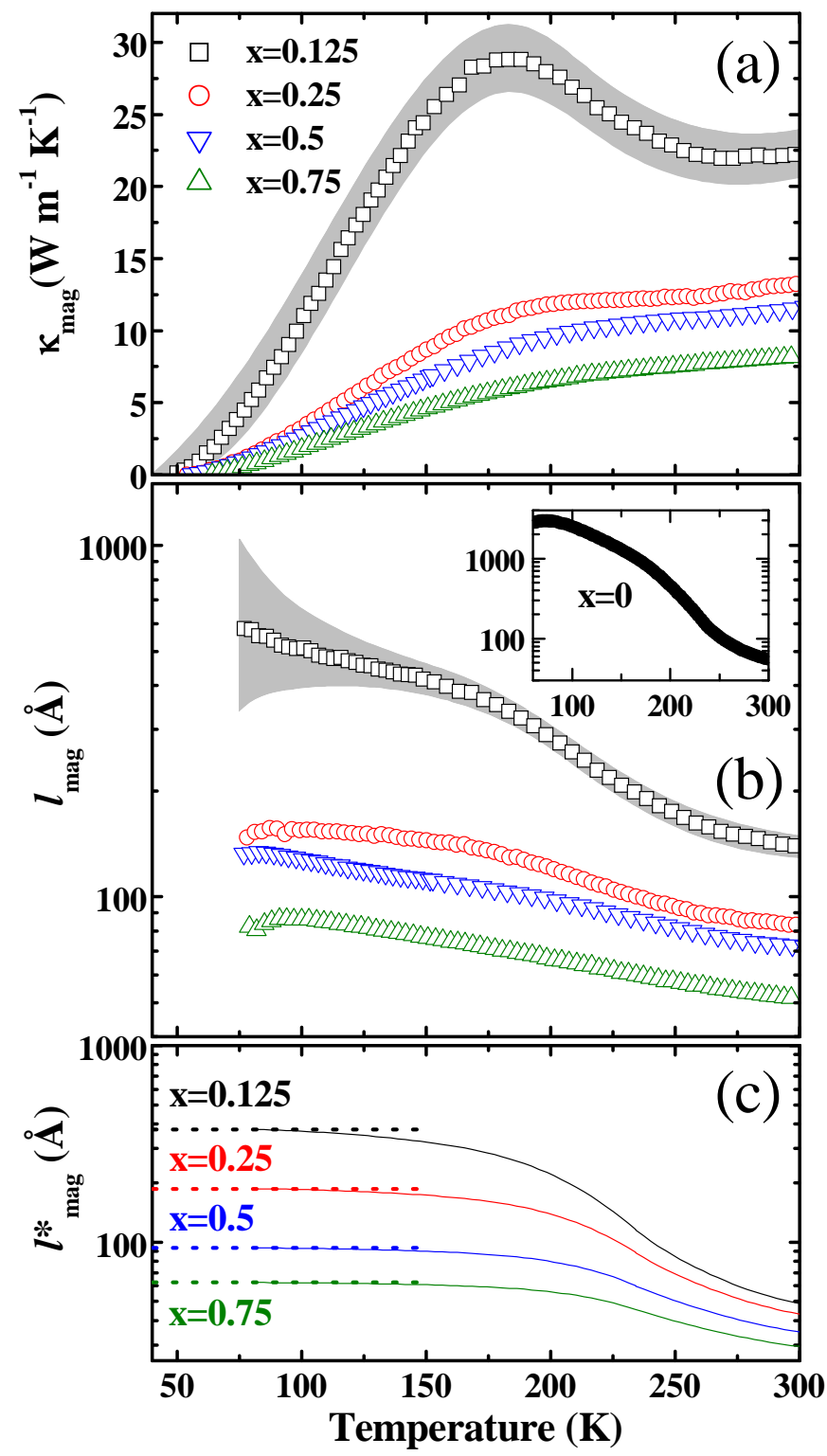

FIG. 2: $\kappa_{\text {mag }}$ (a) and $l_{\text {mag }}$ (b) of $\mathrm{Sr}_{14} \mathrm{Cu}_{24-\mathrm{x}} \mathrm{Zn}_{\mathrm{x}} \mathrm{O}_{41}$ at $x=0.125,0.25,0.5,0.75$ and $x=0$ (Inset). In (b) data points below $75 \mathrm{~K}$ are omitted, because the experimental errors are to large in this temperature range. The grey shaded areas in (a) and (b) display the experimental uncertainty of $\kappa_{\text {mag }}$ and $l_{\text {mag }}$ resulting from the uncertainties of $\kappa_{\mathrm{ph}}$ for the representative case $x=0.125$. The solid lines in (c) represent the estimated $l_{\text {mag }}^{*}(\mathrm{cf}$. text). The dotted lines mark the mean distance of $\mathrm{Zn}$-ions in the ladders.

accordingly upon doping, since $\kappa_{\text {mag }}$ decreases and the peak-like structure vanishes at $x \gtrsim 0.25$.

We proceed now with a quantitative analysis of $\kappa_{\text {mag }}$, where our main interest lies in a comparison of the mean distance of $\mathrm{Zn}$-ions $d_{\mathrm{Zn}-\mathrm{Zn}}$ and $l_{\mathrm{mag}}$ extracted from the data. The analysis is based on a kinetic description of the heat transport in the ladders presented earlier in Ref. 2,

$$
\kappa_{\mathrm{mag}}=\frac{3 N l_{\mathrm{mag}}}{\pi \hbar k_{B} T^{2}} \int_{\Delta}^{\epsilon_{\max }} \frac{\exp \left(\epsilon / k_{B} T\right)}{\left(\exp \left(\epsilon / k_{B} T\right)+3\right)^{2}} \epsilon^{2} d \epsilon .
$$

Here, $N$ is the number of ladders per unit area; $l_{\text {mag }}$, $\Delta$ and $\epsilon_{\max } \approx 200 \mathrm{meV}^{30}$ are the mean free path, the gap and the band maximum of the spin excitations respectively. Presuming that the spin gap ${ }^{2} \Delta=396 \mathrm{~K}$ of undoped $\mathrm{Sr}_{14} \mathrm{Cu}_{24} \mathrm{O}_{41}$ does not significantly change at these light Zn-doping levels, $\stackrel{37}{\stackrel{3}{m}} l_{\text {mag }}$ can directly be calculated from our $\kappa_{\text {mag }}$ data as shown in Fig. 2 p.

Similar to $l_{\mathrm{mag}}(T)$ at $x=0$ (cf. inset of Fig. 2), $l_{\mathrm{mag}}$ of the Zn-doped samples clearly decreases upon heating. The decrease itself, however, is much weaker for the doped samples, and apparently weakens further with increasing Zn-content. In Ref. 5 it has been argued that in the undoped compound the temperature dependence of $l_{\mathrm{mag}}$ is intimately related to temperature dependent magnon-hole scattering which arises due to changes of hole mobility upon charge ordering: at $T \lesssim 100 \mathrm{~K}$, i.e. deep in the charge ordered state, magnon hole scattering is unimportant. It becomes increasingly dominant at higher temperature and reaches its full strength at $T \gtrsim 240 \mathrm{~K}$, i.e. above the charge ordering temperature. $l_{\text {mag }}$ is thereby reduced from $l_{0} \approx 3000 \AA$ at $T \lesssim 100 \mathrm{~K}$ to around $60 \AA$ at $300 \mathrm{~K}$.

Following Matthiesen's rule it is possible to separate $l_{\mathrm{mag}}^{-1}=l_{0}^{-1}+l_{h}^{-1}$ into contributions due to scattering off static defects, $l_{0}$, and off mobile holes, $l_{h}$ 产 The observed reduction of $l_{\text {mag }}$ upon $\mathrm{Zn}$-doping is indeed consistent with a gradually reduced $l_{0}$ due to scattering of magnons on Zn-ions and only a slight change in $l_{h}$. In order to illustrate this, we estimate the mean free path of the Zn-doped samples under simplified conditions, i.e. we assume that firstly $l_{0}=d_{\mathrm{Zn}-\mathrm{Zn}}$ and secondly that $l_{h}(T)$ is the same as in the non-doped material as published

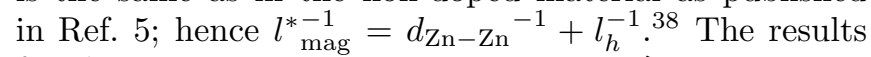
for $l_{\text {mag }}^{*}$ are displayed in Fig. 22. For $T \gtrsim 100 \mathrm{~K}$ there is a striking similarity in both magnitude and temperature dependence between the results of the experimental $l_{\text {mag }}$ and our estimated $l_{\text {mag }}^{*}$ values. We should mention the unavoidable uncertainties in $l_{\text {mag }}$ which are large at $T \lesssim 100 \mathrm{~K}$, since the ratio $\kappa_{\mathrm{mag}} / \kappa_{\mathrm{ph}}$ is small here and errors in the estimation of $\kappa_{\mathrm{ph}}$ have large effects on the estimations of $\kappa_{\text {mag }}$ and $l_{\text {mag }}$ (cf. Fig. 2 2 and 2 2 ). A reliable determination of the experimental $l_{\text {mag }}$ can therefore only be expected at higher temperature.

However, at $100 \mathrm{~K} \lesssim T \lesssim 125 \mathrm{~K}$ such errors become reasonably small and simultaneously $l_{\text {mag }} \approx d_{\mathrm{Zn}-\mathrm{Zn}}$ should hold. (Note that in this temperature range the deviation of $l_{\mathrm{mag}}^{*}$ from $l_{0}=d_{\mathrm{Zn}-\mathrm{Zn}}$ is smaller than $10 \%$, as can be inferred from the dotted lines in Fig. 22.) We therefore test our experimental $l_{\text {mag }}$ from Fig. 2 $2 \mathrm{~b}$ for such a linear scaling at a representative $T=120 \mathrm{~K}$ in Fig. 3. As is evident from the figure, $l_{\text {mag }}$ roughly scales with $d_{\mathrm{Zn}-\mathrm{Zn}}$ and a linear fit to the data points yields a slope of $1.17 \pm 0.25$, which is close to unity. This confirms unambiguously that the values for $l_{\text {mag }}$ obtained from the 


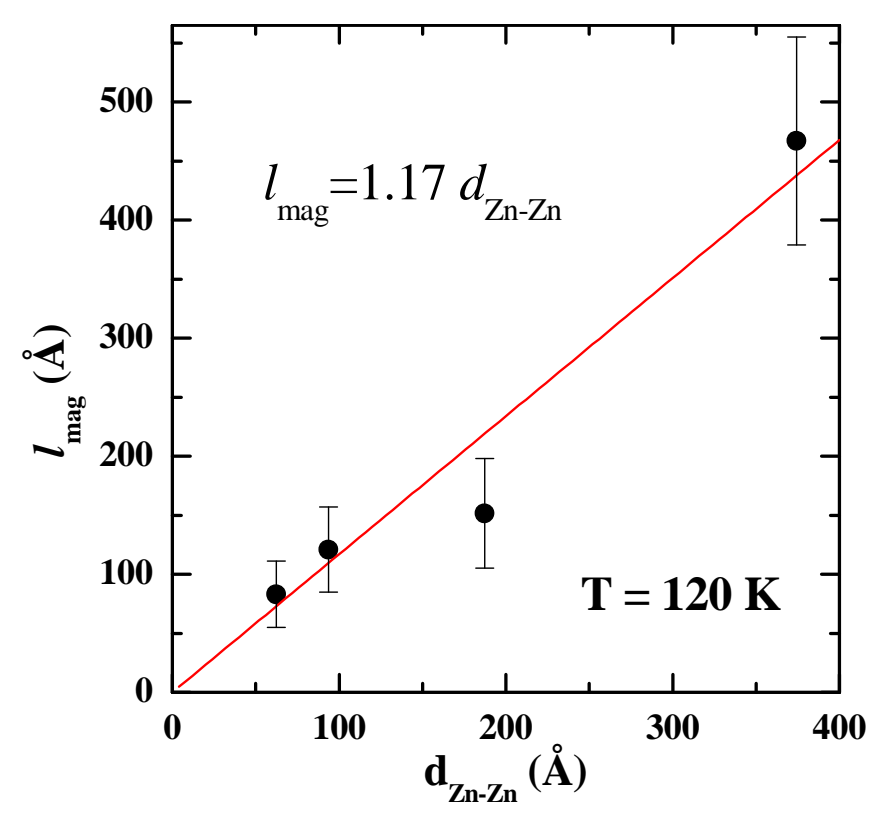

FIG. 3: $l_{\text {mag }}$ as a function of $d_{\mathrm{Zn}-\mathrm{Zn}}$. Solid line: linear fit line through the origin. The error bars arise due to uncertainties in determining $\kappa_{\mathrm{ph}}$ and have been obtained by using different estimates for $\kappa_{\mathrm{ph}}$ (cf. inset of Fig. 11) and Fig. 20.

kinetic model are in fair agreement with real scattering lengths in the material.

\section{DISCUSSION}

The consequences of the analysis outlined above are evident. It is natural to conclude that the kinetic model yields realistic numbers for $l_{\text {mag }}$, not only in the $Z$ n-doped material discussed above but also in $\mathrm{Sr}_{14} \mathrm{Cu}_{24} \mathrm{O}_{41}$ and $\mathrm{La}_{5} \mathrm{Ca}_{9} \mathrm{Cu}_{24} \mathrm{O}_{41}$ where it yields the aforementioned surprising high values of $l_{\text {mag }} \approx 3000 \AA$ at low temperature. Therefore, the rather small values for $l_{\text {mag }}$, which were obtained under the assumption of a finite thermal Drude weight ${ }^{11}$ become less realistic. This is further reinforced by recent theoretical investigations which suggest a vanishing thermal Drude weight ${ }^{19.23}$.

We point out that on a microscopic level the mech- anism of magnon-scattering off nonmagnetic $\mathrm{Zn}^{2+}$ ions and its relevance for magnetic heat transport remains unaddressed. Qualitatively this scattering appears to be very similar to the $2 \mathrm{D}$ case of $\mathrm{La}_{2} \mathrm{CuO}_{4}$, where $l_{\text {mag }} \approx d_{\mathrm{Zn}-\mathrm{Zn}}$ has been observed as well (with $d_{\mathrm{Zn}-\mathrm{Zn}}$ being the mean unidirectional distance of $\mathrm{Zn}$-impurities in the $\mathrm{CuO}_{2}$-planes) $\stackrel{8}{-}$ In this regard it seems to be highly intriguing to investigate the heat transport of Zn-doped spin chain materials such as $\mathrm{SrCuO}_{2}$ and $\mathrm{Sr}_{2} \mathrm{CuO}_{3}$, since Zn-impurities must lead to complete chain interruptions in these cases which could - compared to two-leg ladders and 2D antiferromagnetic planes - result in a qualitatively different effect on $\kappa_{\text {mag }}$.

\section{SUMMARY}

Our experimental study of $\kappa_{\text {mag }}$ of $\mathrm{Sr}_{14} \mathrm{Cu}_{24-\mathrm{x}} \mathrm{Zn}_{\mathrm{x}} \mathrm{O}_{41}$ shows that Zn-doping in this spin ladder compound strongly suppresses $\kappa_{\text {mag }}$ with a progressive decrease upon increasing Zn-content. Since the Zn-ions create defects with a well defined distance the data provide a clearcut and model-independent relation between $\kappa_{\text {mag }}$ and the mean free path $l_{\text {mag }} \lesssim 374 \AA$. This strongly corrob-

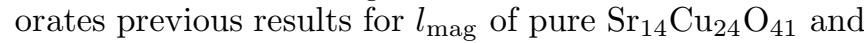
$\mathrm{La}_{5} \mathrm{Ca}_{9} \mathrm{Cu}_{24} \mathrm{O}_{41}$, in which surprisingly large values for $l_{\text {mag }}$ have been found on basis of kinetic mode ${ }^{2}$. We further find that the $l_{\text {mag }}$ determined from the present data with this kinetic model are roughly equal to the mean distance of Zn-ions along the ladders. Magnetic scattering lengths in the spin ladder material $(\mathrm{Sr}, \mathrm{Ca}, \mathrm{La}){ }_{14} \mathrm{Cu}_{24} \mathrm{O}_{41}$ can therefore conveniently be deduced from experimental data for $\kappa_{\text {mag }}$.

\section{Acknowledgments}

This work has been supported by the DFG through SP1073. It is a pleasure to thank W. Brenig, F. HeidrichMeisner, A. Rosch and A.V. Sologubenko for stimulating discussions. We are in particular grateful to F. Heidrich-Meisner and R. Klingeler for carefully reading the manuscript and useful comments. We further thank A.P. Petrovic and A. Waske for proofreading the manuscript.
* c.hess@ifw-dresden.de

1 A. V. Sologubenko, K. Giannò, H. R. Ott, U. Ammerahl, and A. Revcolevschi, Phys. Rev. Lett. 84, 2714 (2000).

2 C. Hess, C. Baumann, U. Ammerahl, B. Büchner, F. Heidrich-Meisner, W. Brenig, and A. Revcolevschi, Phys. Rev. B 64, 184305 (2001).

${ }^{3}$ K. Kudo, S. Ishikawa, T. Noji, T. Adachi, Y. Koike, K. Maki, S. Tsuji, and K. Kumagai, J. Phys. Soc. Jpn. 70, 437 (2001).

${ }^{4}$ C. Hess, U. Ammerahl, C. Baumann, B. Büchner, and A. Revcolevschi, Physica B 312-313, 612 (2002).
${ }^{5}$ C. Hess, H. ElHaes, B. Büchner, U. Ammerahl, M. Hücker, and A. Revcolevschi, Phys. Rev. Lett. 93, 027005 (2004).

${ }^{6}$ A. V. Sologubenko, E. Felder, K. Giannò, H. R. Ott, A. Vietkine, and A. Revcolevschi, Phys. Rev. B 62, R6108 (2000).

7 A. V. Sologubenko, K. Giannò, H. R. Ott, A. Vietkine, and A. Revcolevschi, Phys. Rev. B 64, 054412 (2001).

8 C. Hess, B. Büchner, U. Ammerahl, L. Colonescu, F. Heidrich-Meisner, W. Brenig, and A. Revcolevschi, Phys. Rev. Lett. 90, 197002 (2003).

9 M. Hofmann, T. Lorenz, K. Berggold, M. Grüninger, 
A. Freimuth, G. S. Uhrig, and E. Brück, Phys. Rev. B 67, 184502 (2003).

10 X. Zotos, F. Naef, and P. Prelovsek, Phys. Rev. B 55, 11029 (1997).

11 J. V. Alvarez and C. Gros, Phys. Rev. Lett. 89, 156603 (2002).

12 A. Klümper and K. Sakai, J. Phys. A: Math. Gen. 35, 2173 (2002).

13 K. Sakai and A. Klümper, J. Phys. A: Math. Gen. 36, 11617 (2003).

${ }^{14}$ F. Heidrich-Meisner, A. Honecker, D. C. Cabra, and W. Brenig, Phys. Rev. B 66, 140406(R) (2002).

15 F. Heidrich-Meisner, A. Honecker, D. C. Cabra, and W. Brenig, Phys. Rev. B 68, 134436 (2003).

16 E. Shimshoni, N. Andrei, and A. Rosch, Phys. Rev. B 68, 104401 (2003).

17 A. V. Sologubenko, S. M. Kazakov, H. R. Ott, T. Asano, and Y. Ajiro, Phys. Rev. B 68, 094432 (2003).

18 A. V. Sologubenko, H. R. Ott, G. Dhalenne, and A. Revcolevschi, Europhys. Lett. 62, 540 (2003).

19 X. Zotos, Phys. Rev. Lett. 92, 067202 (2004).

${ }^{20}$ K. Saito, Phys. Rev. B 67, 064410 (2003).

21 E. Orignac, R. Chitra, and R. Citro, Phys. Rev. B 67, 134426 (2003).

22 F. Meier and D. Loss, Phys. Rev. Lett. 90, 167204 (2003).

23 F. Heidrich-Meisner, A. Honecker, D. C. Cabra, and W. Brenig, Phys. Rev. Lett. 92, 069703 (2004).

${ }^{24}$ F. Heidrich-Meisner, A. Honecker, and W. Brenig, Phys. Rev. B 71, 184415 (2005).

25 A. V. Rozhkov and A. L. Chernyshev, Phys. Rev. Lett. 94, 087201 (2005).

26 A. L. Chernyshev and A. V. Rozhkov, Phys. Rev. B 72, 104423 (2005).

27 U. Ammerahl, G. Dhalenne, A. Revcolevschi, J. Berthon, and H. Moudden, J. Crystal Growth 193, 55 (1998).

28 C. Hess, B. Büchner, M. Hücker, R. Gross, and S.-W. Cheong, Phys. Rev. B 59, R10397 (1999).

29 C. Hess, B. Büchner, U. Ammerahl, and A. Revcolevschi, Phys. Rev. B 68184517 (2003).
30 R. S. Eccleston, M. Uehara, J. Akimitsu, H. Eisaki, N. Motoyama, and S. I. Uchida, Phys. Rev. Lett. 81, 1702 (1998).

31 S. Katano, T. Nagata, J. Akimitsu, M. Nishi, and K. Kakurai, Phys. Rev. Lett. 82, 636 (1999).

32 J. Callaway and H. C. von Baeyer, Physical Review 120, 1149 (1960).

33 M. Azuma, Y. Fujishiro, M. Takano, M. Nohara, and H. Takagi, Phys. Rev. B 55, R8658 (1997).

34 We note that at all doping levels we observed a tendency of $\kappa_{c}$ to vary from measurement to measurement. Similar behavior has frequently been found in other quasi onedimensional spin systems and will be discussed elsewhere. Nevertheless, we stress that all data presented here were reproducible in two subsequent measurements.

35 For the fit we used ${ }^{32} \kappa_{\mathrm{ph}}=\frac{k_{B}}{2 \pi^{2} v}\left(\frac{k_{B} T}{\hbar}\right)^{3} \int_{0}^{\Theta_{D} / T} \frac{\tau_{c} x^{4} e^{x}}{\left(e^{x}-1\right)^{2}} d x$ with the scattering rate $\tau_{c}^{-1}=A \omega^{4}+v / L+$ $B T \omega^{3} \exp \left(-\Theta_{D} / b T\right)$, where $\omega, \Theta_{D}$ and $v$ are the phonon frequency, Debye temperature and mean phonon velocity. The three terms correspond to scattering due to defects, crystal boundaries and umklapp processes respectively. $A$, $L, B$ and $b$ are free fit parameters with the constraint that $L, B$ and $b$ are identical for all $\mathrm{Zn}$-doped samples.

36 The different phononic backgrounds have been obtained from fitting data points at $T \leq(53 \pm 12) \mathrm{K}$.

37 Despite other Zn-doped low-dimensional quantum spins systems ${ }^{33}$ our data on $\mathrm{Sr}_{14} \mathrm{Cu}_{24-\mathrm{x}} \mathrm{Zn}_{\mathrm{x}} \mathrm{O}_{41}$ do not yield any evidence for magnetic order at low temperature.

38 Resistivity data of $\mathrm{Sr}_{14} \mathrm{Cu}_{24-\mathrm{x}} \mathrm{Zn}_{\mathrm{x}} \mathrm{O}_{41}$ indicate a weakening of the charge-ordered state upon $\mathrm{Zn}$-doping. An additional suppression of $l_{\text {mag }}$ could therefore arise due to an increased mobility of holes in the ladders and a thereby reduced $l_{h}$. However, this effect appears to be only of minor importance, since a clear signature of charge ordering is still observed at the lowest doping level $x=0.125$, whereas $l_{\text {mag }}$ is already strongly suppressed. In any case, the main doping- and temperature dependent features of $l_{\mathrm{mag}}^{*}$ are quite robust against such doping dependencies as long as $l_{h} \gg l_{0}$ at low temperature. 